\title{
The silver effect of admission glucose level on excellent outcome in thrombolysed stroke patients
}

\author{
Charlotte Rosso ${ }^{1,2}\left(\right.$ D . Flore Baronnet ${ }^{2} \cdot$ Belen Diaz $^{2} \cdot$ Raphael Le Bouc $^{1,2} \cdot$ Giulia Frasca Polara ${ }^{2} \cdot$ Eric Jr Moulton ${ }^{1}$. \\ Sandrine Deltour ${ }^{2} \cdot$ Anne Leger $^{2} \cdot$ Sophie Crozier $^{2} \cdot$ Yves Samson $^{1,2}$
}

Received: 28 March 2018 / Revised: 2 May 2018 / Accepted: 3 May 2018 / Published online: 18 May 2018

(C) The Author(s) 2018

\begin{abstract}
Higher admission glucose levels (AGL) are associated with less favorable outcome in thrombolysis. But, could AGL's impact on outcome vary by onset-to-treatment (OTT) time? Is hyperglycemia associated with a shorter therapeutic time window for excellent outcome for thrombolysed stroke patients? We assessed predictive values of AGL, baseline NIHSS, age, and OTT time quartiles on excellent outcome (3-month modified Rankin score of 0-1) in 773 patients treated by rt-Pa. We added the AGL $\times$ OTT time quartile interaction in the model and separately analyzed the predictive values of AGL, age, and NIHSS for each OTT time quartile if the interaction was significant. AGL, baseline NIHSS, age, and OTT time quartiles were significant predictors. When added in the model, the AGL $\times$ OTT interaction was significant (OR: 0.96, 95\% CI: $0.94-0.99, p: 0.0009)$. AGL was predictive only during the third OTT time quartile (181-224 min). During this period, the predicted rate of excellent outcome was $16 \%$ for $\mathrm{AGL}=6.5 \mathrm{mmol} / \mathrm{L}$ and $8 \%$ for $\mathrm{AGL}=8 \mathrm{mmol} / \mathrm{L}$. The rate of excellent outcome was not decreased in hyperglycemic patients for OTT time $\leq 180 \mathrm{~min}$ ( $20 \mathrm{vs.} 24.5 \% p$ : 0.37), but was decreased for OTT time $>180 \mathrm{~min}$ (9.6 vs. $26.7 \%$ p: 0.00001). Similar results were found in patients with MCA recanalization, but not in patients without recanalization. The therapeutic time window for excellent outcome is shortened in hyperglycemic patients. This would support the design of "freezing penumbra" randomized trials based on ultra-early AGL control.
\end{abstract}

Keywords Acute stroke $\cdot$ Hyperglycemia $\cdot$ Cohort studies $\cdot$ Prognosis

\section{Introduction}

In the era of recanalization therapies, the factors responsible for the onset-to-treatment (OTT) time vs. outcome relationship need further investigations in acute stroke patients. Treating the factors that shorten the therapeutic time window for excellent outcome in fact may correspond to slow down the penumbra's evolution. Baseline post-stroke hyperglycemia could be one of these factors. To date, most studies showed that higher admission glucose levels (AGL) were

Charlotte Rosso

charlotte.rosso@gmail.com

1 Inserm U 1127, CNRS UMR 7225, UMR S 1127, Institut du Cerveau et de la Moelle épinière, ICM, Sorbonne Université, 75013 Paris, France

2 APHP, Urgences Cérébro-Vasculaires, Hôpital Pitié-Salpêtrière, 47-83 Boulevard de l'Hôpital, 75013 Paris, France associated with less favorable outcome in thrombolysis [1], but could AGL's impact on outcome vary by OTT time?

This possibility arises from an association between hyperglycemia and an accelerated infarct growth in experimental and imaging studies as reviewed in Piironen et al. [2]. Moreover, in these studies, there is accumulating evidence indicating that the glucose toxicity threshold was low (between 6 and $8 \mathrm{mmol} / \mathrm{l}$ ) [3]. To our knowledge, the interaction between OTT time and AGL has never been formally tested.

The proportion of patients with excellent outcome after thrombolysis sharply decreases when a threshold volume of infarction is reached, corresponding to a certain amount of penumbra volume being transformed. Parsons et al. reported that $48 \%$ of patients with diffusion-weighted imaging (DWI) lesions $<18 \mathrm{ml}$ at admission had excellent outcome at 3 months, compared to only $15 \%$ with DWI lesions $>18 \mathrm{ml}$ [4], with similar results reported by others [5-7]. Our hypothesis is that the true effect of OTT time on infarct volume may actually be changed by a penumbra-modifying factor, such as AGL (Fig. 1). More precisely and theoretically 
Fig. 1 The silver effect

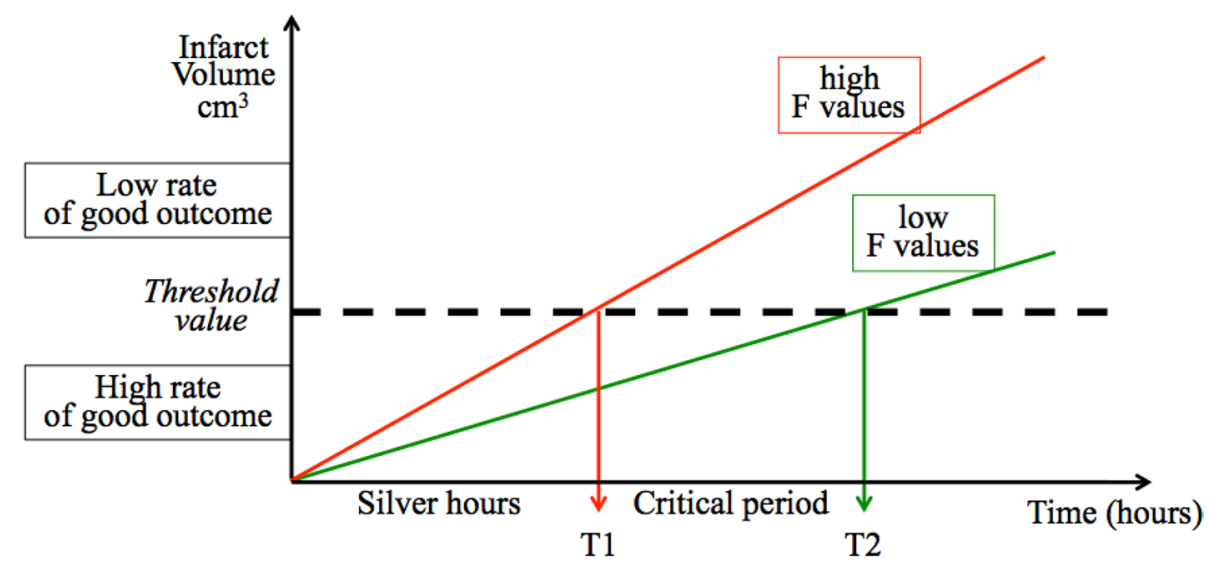

(Fig. 1), if a factor $F$ (i.e., a penumbra-modifying factor, such as AGL) increases the rate of infarct growth, the infarct volume threshold for excellent outcome will be reached at an earlier time (T1) for higher values of $F$. Conversely, lower values of $F$ can extend this cut-off point to a later time (T2). Prior to T1, the rate of excellent outcome will be high for any value of $F$, and $F$ will not statistically predict outcome. We called this period (before T1) the "silver hours", by analogy with the golden hour [8]. During the subsequent "critical period" (T1-T2), the rate of excellent outcome will decrease faster for higher values of $F$, which will ultimately predict outcome. Beyond $\mathrm{T} 2$ or in the absence of recanalization, the rate of excellent outcome will continue to decrease even for low values of $F$, which will lose its predictive value. In other words, a "silver effect" predicts a significant interaction between OTT time and $F$, with the predictive value of $F$ for excellent outcome being low during the silver hours, high during the critical T1-T2 period, and low again beyond T2. A "silver effect" also implies that the OTT time $\times F$ interaction should be significant in patients with successful thrombolysis but not in those with persistent occlusion. In this latter, time goes on beyond T2 and, whatever the level of $F$, patients are more likely to have a poor outcome as the infarct volume will grow up to the critical threshold.

We investigated this issue in our prospective registry of patients treated by intravenous thrombolysis. We hypothesized that AGL at admission could constitute a hypothetical factor $F$ and tested the interaction between AGL and OTT time on outcome.

\section{Methods}

\section{Patients}

We used the data of the Pitie-Salpêtrière prospective registry of patients treated by intravenous rtPA for an anterior circulation ischemic stroke. Between February 2000 and February 2016, 904 consecutive patients were treated. According to the routine clinical protocol at our institution, we treated the patients within a 4.5 -h time window with the usual dose $(0.9 \mathrm{mg} / \mathrm{kg}$ for a maximal dose of $90 \mathrm{mg})$. Symptom onset was defined as the last time period that the patient had exhibited normal health. Admission serum glucose level was measured before any treatment and available in 890 patients $(98.5 \%$ ) in the registry. A neurological examination was assessed using the National Institute of Health Stroke Scale (NIHSS) at admission (prior to the magnetic resonance imaging-MRI and any treatment) and on days one (D1) and seven (D7). Modified Rankin scores (mRs) were assessed at 3 months in routine and available in the registry for 773 patients $(86.9 \%)$.

\section{Data analysis}

Descriptive statistics consisted of median and interquartile ranges (IQR). Comparisons of proportions were determined by a Chi-squared test.

\section{Impact of AGL and OTT time on excellent outcome}

We used the 3-month mRs of 0 or 1 as a primary outcome criterion for excellent outcome. Since NIHSS, age, OTT time, and AGL are robust predictors of outcome in most studies, we first investigated their univariate and then their multivariate predictive values. OTT time was divided into quartiles. The multivariate analysis was done using stepwise logistic regression, the variables being entered in the final model at $p \leq 0.05$ and removed at $p>0.10$.

To test the "silver effect" hypothesis, we then added in the model the OTT time quartile $\times$ AGL interaction. In the case of a significant interaction, we planned to run stepwise logistic regressions in the four OTT time quartiles using age, NIHSS, and AGL as independent variables. When AGL was a significant predictor, we calculated the best 
sensitivity-specificity threshold of AGL associated with excellent outcome using ROC curve analysis.

As stated in the introduction, a "silver effect" also implies that the OTT time $\times$ AGL interaction should be significant in patients with successful thrombolysis (i.e., recanalization) but not in those with persistent occlusion. We therefore compared the results in subgroups of patients with and without complete middle cerebral artery recanalization. Intracranial occlusion and arterial recanalization were assessed on initial $(<6 \mathrm{~h})$ and follow-up MR interpretable angiography (at 24 h), respectively ( $n=724,94 \%$ and $n=658,85 \%$ ). Recanalization was considered on a 3-item scale: (1) patent or complete recanalization; (2) partial or minimal flow-related signal in the region of the arterial clot; and (3) persistent occlusion.

\section{Impact of AGL and OTT time on good outcome}

The analyses were additionally performed on 3-month mRs scores of $0-2$ to verify that good outcome is also potentially affected by a "silver effect".

\section{Results}

\section{Patients}

Among the 773 included patients, 52.5\% $(n=406)$ were men. Thirty-six patients were additionally treated by endovascular treatment (4.6\%). The median and interquartile (IQR) values of admission NIHSS was 16 (11-21), age: 67 years (53-80 years), AGL: 6.5 (5.8-7.8) $\mathrm{mmol} / \mathrm{L}$, and OTT time:
$181 \min (142-225 \mathrm{~min})$. The 131 patients excluded for missing data were comparable to those included for baseline NIHSS (median NIHSS: 16 vs. $16, p$ : 0.44 ) but were older than the included ones (median age: 74 vs. 67 years, p: 0.002). Day-one median NIHSS was 12 (IQR: 7-19) and day-seven NIHSS was 8 (IQR: 2-13). At 3 months, excellent outcome (mRs $0-1)$ was achieved in $20.2 \%(n=156)$ of patients, good outcome (mRs $0-2)$ in $40.2 \%(n=311)$, and the death rate was $17.1 \%(n=132)$.

At admission, intracranial MRA revealed MCA trunk (M1) occlusions in 17\% $(n=125)$ of patients, MCA branches (M2) occlusions in $40 \%(n=292)$, intracranial occlusions of the carotid artery in $29 \%(n=211)$, miscellaneous occlusions in $4.5 \%(n=34)$, and no occlusions in $9.5 \%(n=62)$. At $24 \mathrm{~h}$, the MCA was patent in $46.6 \%(n=306)$ of cases, partially recanalized in $24 \%(n=158)$, and remained occluded in $29.4 \%(n=194)$. Symptomatic hemorrhage occurred in $7.5 \%(58 / 765)$.

The characteristics of the patients sorted in the four OTT time quartiles (<142 min; 142-180; 181-224; > $224 \mathrm{~min})$ are shown in Table 1 . The four groups were similar except that patients in the first quartile were older than those of the fourth quartile ( $p: 0.002)$. The rate of excellent outcome decreased significantly from $25.5 \%$ in the first OTT time quartile, to $16.4 \%$ in the last OTT time quartile ( $p$ : 0.03$)$.

\section{Prediction of excellent outcome ( $m R s ~ 0-1)$}

NIHSS, age, OTT time quartile, and AGL were significant predictors in the univariate analysis and remained significant in the multivariate stepwise logistic regression (Table 2). When the OTT time quartile*AGL interaction was

Table 1 Characteristics of the patients in the four OTT quartiles

\begin{tabular}{llllll}
\hline OTT quartiles & OTT time $(\min )$ & Baseline NIHSS & AGL (mmol/l) & Age (years) & mRs 0-1 (\%) \\
\hline 1st; $<142 \min (\mathrm{n}=193)$ & $120(109-139)$ & $16(11-22)$ & $6.5(5.8-7.7)$ & $70.5(55.7-81.9)$ & 25.5 \\
2nd; $142-180 \min (\mathrm{n}=192)$ & $163(152-175)$ & $17(11-22)$ & $6.5(5.8-8)$ & $66.9(53.8-81.3)$ & 19.7 \\
3rd; $181-224 \min (\mathrm{n}=193)$ & $200(190-210)$ & $16(11-21)$ & $6.5(5.7-7.7)$ & $66.3(53.9-80)$ & 19.7 \\
4th; $>224 \min (\mathrm{n}=195)$ & $260(240-284)$ & $15(10-20)$ & $6.6(5.8-8.3)$ & $63.2(49.5-77.2)$ & 16.4 \\
\hline
\end{tabular}

Values are median and IQR

Table 2 Predictive values of AGL, OTT time quartiles, baseline NIHSS, and age on 3 months excellent outcome $(\mathrm{mRS} 0-1)$

\begin{tabular}{|c|c|c|c|c|}
\hline & \multicolumn{2}{|l|}{ Univariate analysis } & \multicolumn{2}{|c|}{ Multivariate analysis } \\
\hline & OR, $95 \%$ CI & $p$ & OR, 95\% CI & $p$ \\
\hline AGL (mmol/l) & $0.84(0.76-0.93)$ & 0.001 & $0.89(0.80-0.98)$ & 0.02 \\
\hline OTT time quartile & $0.85(0.72-0.99)$ & 0.04 & $0.77(0.65-0.92)$ & 0.004 \\
\hline Baseline NIHSS & $0.84(0.81-0.87)$ & 0.00001 & $0.84(0.81-0.87)$ & 0.00001 \\
\hline Age & $0.98(0.97-0.99)$ & 0.0002 & $0.98(0.97-0.99)$ & 0.001 \\
\hline
\end{tabular}

The multivariate analysis is the final model of the stepwise logistic regression when the four variables were entered without entering the AGL $\times$ OTT time quartile interaction term 
introduced in the logistic regression, the final model retained three independent predictors: NIHSS (OR: 0.84, 95\% CI: 0.81-0.87, $p<0.00001$ ), age (OR: 0.98, 95\% CI: 0.97-0.99, $p=0.002$ ) and the OTT time quartile*AGL interaction (OR: 0.96, 95\% CI: 0.94-0.99, $p=0.0009)$.

Since the interaction term was significant, we analyzed the predictive values of AGL in the four OTT time quartiles. AGL was a significant predictor only in the third OTT time quartile (181-224 min), with a 52\% decreased probability of excellent outcome per mmol/L increase (OR: 0.48; $95 \%$ CI: $0.33-0.70, p<0.0001)$. The ROC curve analysis of AGL predicting 3-month excellent outcome was also significant only in the third OTT time quartile. The AUC was $0.73(95 \%$ CI: $0.67-0.79, p$ : 0.0001$)$, corresponding to good diagnostic performance [9]. The best compromise between sensitivity and specificity to predict excellent outcome was for a threshold of AGL $\leq 6.5 \mathrm{mmol} / \mathrm{l}$, yielding a sensitivity of $81.6 \%$ (95\% CI: 65.7-92.2) and a specificity of 56.8\% (95\% CI: 48.6-64.7). The low specificity, however, indicated that the false positive rate was high (patients with low AGL and poor outcome).

The final multivariate logistic regression models are shown in Table 3 . These models predicted a rate of excellent outcome for a "median" patient $(\mathrm{NIHSS}=16,67$ years old, $\mathrm{AGL}=6.5 \mathrm{mmol} / \mathrm{L}(118 \mathrm{mg} / \mathrm{dL}))$ of $26 \%$ during the first quartile (OTT time $<142 \mathrm{~min}$ ), 16\% during the second and third quartile, and 10\% during the last quartile (OTT time $>224 \mathrm{~min})$. AGL was an independent predictor only during the third quartile (181-224 min). During this "critical period", the predicted rate of excellent outcome decreased from $16 \%$ for $\mathrm{AGL}=6.5 \mathrm{mmol} / \mathrm{l}(118 \mathrm{mg} / \mathrm{dL})$ to $8 \%$ for 8 $\mathrm{mmol} / \mathrm{l}(145 \mathrm{mg} / \mathrm{dL})$.

As expected, the rate of excellent outcome differed in patients with and without complete MCA recanalization (32.9 vs. $11.9 \%, p<0.0001)$. In patients with MCA recanalization, the multivariate logistic regression retained the same variables as in the complete analysis: OTT time quartiles*AGL interaction (OR: 0.96; 95\% CI: 0.93-0.98, $p=0.001$ ), age (OR: 0.98; 95\% CI: 0.96-0.99, $p=0.004)$, and NIHSS (OR: 0.89; 95\% CI: 0.85-0.93, $p<0.00001$ ). Similar to the complete analysis, the logistic regressions performed with the different quartiles revealed that AGL was a significant predictor only in the third OTT time quartile
(OR: 0.44; 95\% CI: 0.27-0.73, $p=0.001$ ), whereas NIHSS was a significant predictor in the three other quartiles.

In patients without complete recanalization, the multivariate logistic regression retained the baseline NIHSS as a predictor of excellent outcome (OR: 0.78; 95\% CI: 0.73-0.84, $p<0.00001$ ), and hyperglycemia did not affect the rate of excellent outcome.

\section{Prediction of good outcome (mRs 0-2)}

The multivariate prediction of 3 months good outcome ( $\mathrm{mRs}$ 0-2) gave similar results to the prediction of excellent outcome with 3 independent predictors: OTT time*AGL interaction (OR: 0.97, 95\% CI: 0.95-0.98; $p=0.0001)$, age (OR: 0.96, 95\% CI: 0.95-0.98; $p<0.00001$ ), and NIHSS (OR: 0.83, 95\% CI: 0.80-0.85; $p<0.00001)$.

\section{Discussion}

We found that AGL was predictor of excellent and good outcome even after adjustment for NIHSS, age, and OTT time. The results were highly consistent with those of a recent systematic review [1]. The new finding was the existence of a strong interaction between AGL and the OTT time quartile. This indicates that the relation between AGL and excellent outcome varies with OTT time. Further analyses suggested that hyperglycemia was associated with a shorter therapeutic time window for excellent outcome with what we called a "silver effect". In subgroup analyses, these findings were only observed in the case of complete MCA recanalization.

\section{Hyperglycemia decreases the time window for excellent outcome after thrombolysis}

AGL was a significant predictor only during the third quartile (181-224 min). In this critical period, the predicted rate of excellent outcome decreased from 16 to $8 \%$ when, for example, AGL increased from 6.5 to $8 \mathrm{mmol} / \mathrm{L}$ (118 to $145 \mathrm{mg} / \mathrm{dL})$. A cutoff AGL of $6.5 \mathrm{mmol} / \mathrm{L}$ was identified by the ROC curve analysis, close to the $6.8 \mathrm{mmol} / \mathrm{L}(123 \mathrm{mg} /$ $\mathrm{dL})$ value reported for good outcome in the SITS-ISTR register [10]. Using the $6.5 \mathrm{mmol} / \mathrm{l}$ cutoff, the rate of excellent outcome was not affected by hyperglycemia when OTT time

Table 3 Final model of the multivariate logistic regression in the four OTT quartiles for excellent outcome

\begin{tabular}{lllll}
\hline OTT time quartiles & $<142 \mathrm{~min}$ & $142-180 \mathrm{~min}$ & $181-224 \mathrm{~min}$ & $>224 \mathrm{~min}$ \\
\hline AGL & - & - & $0.57(0.38-0.86) p=0.008$ & - \\
NIHSS & $\begin{array}{c}0.84(0.79-0.90) \\
\quad p=0.00001\end{array}$ & $0.86(0.81-0.92) p=0.00001$ & $0.83(0.77-0.90) p=0.00001$ & $0.83(0.77-0.90) p=0.00001$ \\
Age & - & $0.96(0.94-0.99) p=0.002$ & - & $0.97(0.95-0.998) p=0.04$ \\
\hline
\end{tabular}


was $\leq 3 \mathrm{~h}$ but was markedly decreased by hyperglycemia when OTT time was between 3 and $3 \mathrm{~h} 44 \mathrm{~min}$. This is consistent with the report of Ribo et al. who found that the time to arterial recanalization leading to poor outcome $(\mathrm{mRs}>2)$ was much shorter $(3.5 \mathrm{~h})$ in patients with hyperglycemia than in patients with adequate glucose control $(7.5 \mathrm{~h})$. Moreover, the authors report that, on average, the infarct grew 2.7 times faster in patients with hyperglycemia during arterial occlusion [11]. This is perhaps why ultrasound-enhanced thrombolysis, which speeds up arterial recanalization [12], mitigated the detrimental effect of increasing AGL on good outcome in the CLOTBUST trial [13].

\section{The "silver effect" of AGL}

The "silver effect" implies that the relation between AGL and excellent outcome would be maximal during a critical period of OTT time (between T1 and T2 on Fig. 1), and this should selectively be observed in patients with successful thrombolysis. Our results provide evidence for the existence of a silver effect since the AGL $\times$ OTT time quartile interaction was significant, the negative relation between AGL and outcome was observed only the third OTT quartile, and these findings were selectively found in patients with complete MCA recanalization. The link between the predictive value of AGL on outcome and time to early recanalization has been previously reported by Alvarez-Sabin et al. [14, 15]. However, in these studies, AGL was a predictor even if recanalization occurred $\leq 180 \mathrm{~min}$, whereas in our study, AGL predicted outcome only in the 181-224 min OTT time period. The discrepancy may have several explanations. First, the 180 min "silver hours" in our study is based on the arbitrary division of OTT time in quartiles. Second, Fig. 1 shows that the timing of $\mathrm{T} 1$ and $\mathrm{T} 2$ will depend on the rate of infarct growth, the impact of AGL on infarct growth, and the value of the infarct threshold volume. Since these three parameters are variable, the exact timing of the critical period may differ between studies and may have been shorter in studies by Alvarez-Sabin et al. [14, 15]. This is also consistent with the results of previous MRI-based studies [8, 16-18, 16-18].

\section{General comments and limitations}

Our results indicated that glucose control is unlikely to improve the rate of excellent outcome unless the treatment started less than 2-3 h after stroke onset. This may in part explain the negative results of published insulin trials, which generally included patients $<24 \mathrm{~h}$ post-stroke [19]. Even in INSULINFARCT, which included patients $<6 \mathrm{~h}$ post-stroke onset, only 22 patients were treated within 2 h [20]. The authors also suggested that strict glucose level control, perhaps even above $6.5 \mathrm{mmol} / \mathrm{l}$, should be targeted.
Our results should not be over-interpreted. First, the shorter time window for excellent outcome did not prove that the time window for thrombolysis is shorter in hyperglycemic patients. This would require the demonstration of an AGL $\times$ OTT time $\times$ treatment interaction in a study with control subjects. Of note, in the IST trial, which randomized about three-quarters of the patients after $3 \mathrm{~h}$, the AGL $\times$ treatment interaction was not significant, but AGL values spanning 5-8 $\mathrm{mmol} / \mathrm{L}$ were merged in the same category [21]. Second, the statistical associations described here do not formally prove a causal link between AGL and outcome. This will remain uncertain unless "freezing" penumbra trials turn out to be positive. Third, the "silver effect" itself is an appealing hypothesis, but remains as such since OTT time is an imprecise surrogate marker of infarct growth [22]. Furthermore, the timing of recanalization was unknown, we cannot exclude that it was slowed down. Therefore, it would be of interest to investigate in patients treated with EVT whether there is a significant AGL $\times$ time to reperfusion interaction and if the steep declines in rates of excellent and good outcome with onset to reperfusion times between 190 and $390 \mathrm{~min}$ (efig 5 in Saver et al. [6]) are modified by AGL. This would be an important argument in favor of "freezing the penumbra" therapeutic strategies by ultra-early glycemic control. One experimental study suggests that this approach may be beneficial [23]. Yet, this remains to be proven by randomized trials, since the pathophysiological mechanisms linking hyperglycemia and acute ischemia are complex and still debated [24], and intensive insulin treatment may paradoxically increase infarct growth [20, 25].

\section{Conclusions}

In this study, we highlighted that the relation between AGL and excellent/good outcome varies by OTT time. In other words, hyperglycemia was associated with a shorter therapeutic time window for excellent/good outcome with what we called a "silver effect". These results would support the design of "freezing penumbra" randomized trials based on ultra-early AGL control $(<3 \mathrm{~h})$ targeting nearly normal glycemic levels $(<6.5 \mathrm{mmol} / \mathrm{L}$, i.e., $115 \mathrm{mg} / \mathrm{dL})$.

Acknowledgements This study was supported by the French ministry of health grant EVALUSINV PHRC national 2003 AOM 03008.

Funding The research leading to these results has received funding from the program "Investissements d'avenir" ANR-10-IAIHU-06.

Conflicts of interest The authors declare that they have no conflict of interest.

Ethic statement The Pitié-Salpêtrière registry had approval by an ethics committee when it was created (PHRC AOM 003008, Paris VI ethics committee), but in accordance with French legislation we did 
not need written informed consent from patients, as it is a retrospective database implying only analysis of anonymized data collected prospectively as part of routine clinical care.

Open Access This article is distributed under the terms of the Creative Commons Attribution 4.0 International License (http://creativeco mmons.org/licenses/by/4.0/), which permits unrestricted use, distribution, and reproduction in any medium, provided you give appropriate credit to the original author(s) and the source, provide a link to the Creative Commons license, and indicate if changes were made.

\section{References}

1. Desilles JP, Meseguer E, Labreuche J et al (2013) Diabetes mellitus, admission glucose, and outcomes after stroke thrombolysis: a registry and systematic review. Stroke 44:1915-1923

2. Piironen K, Putaala J, Rosso C, Samson Y (2012) Glucose and acute stroke: evidence for an interlude. Stroke 43:898-902

3. Garg R, Chaudhuri A, Munschauer F, Dandona P (2006) Hyperglycemia, insulin, and acute ischemic stroke: a mechanistic justification for a trial of insulin infusion therapy. Stroke 37:267-273

4. Parsons MW, Christensen S, McElduff P et al (2010) Pretreatment diffusion- and perfusion-MR lesion volumes have a crucial influence on clinical response to stroke thrombolysis. J Cereb Blood Flow Metab 30:1214-1225

5. Ribo M, Tomasello A, Lemus M et al (2015) Maximal admission core lesion compatible with favorable outcome in acute stroke patients undergoing endovascular procedures. Stroke 46:2849-2852

6. Saver JL, Goyal M, van der Lugt A et al (2016) Time to treatment with endovascular thrombectomy and outcomes from ischemic stroke: a meta-analysis. JAMA 316:1279-1288

7. Shimoyama T, Kimura K, Uemura J, Saji N, Shibazaki K (2014) Elevated glucose level adversely affects infarct volume growth and neurological deterioration in non-diabetic stroke patients, but not diabetic stroke patients. Eur J Neurol 21:402-410

8. Saver JL, Smith EE, Fonarow GC et al (2010) The "golden hour" and acute brain ischemia: presenting features and lytic therapy in $>30,000$ patients arriving within 60 minutes of stroke onset. Stroke 41:1431-1439

9. Obuchowski NA (2003) Receiver operating characteristic curves and their use in radiology. Radiology 229:3-8

10. Ahmed N, Davalos A, Eriksson N et al (2010) Association of admission blood glucose and outcome in patients treated with intravenous thrombolysis: results from the Safe Implementation of Treatments in Stroke International Stroke Thrombolysis Register (SITS-ISTR). Arch Neurol 67:1123-1130
11. Ribo M, Molina CA, Delgado P et al (2007) Hyperglycemia during ischemia rapidly accelerates brain damage in stroke patients treated with tPA. J Cereb Blood Flow Metab 27:1616-1622

12. Alexandrov AV, Molina CA, Grotta JC, Garami Z, Ford SR, Alvarez-Sabin J et al (2004) Ultrasound-enhanced systemic thrombolysis for acute ischemic stroke. N Engl J Med 351:2170-2178

13. Martini SR, Hill MD, Alexandrov AV, Molina CA, Kent TA (2006) Outcome in hyperglycemic stroke with ultrasound-augmented thrombolytic therapy. Neurology 67:700-702

14. Alvarez-Sabin J, Molina CA, Montaner J et al (2003) Effects of admission hyperglycemia on stroke outcome in reperfused tissue plasminogen activator-treated patients. Stroke 34:1235-1241

15. Alvarez-Sabin J, Molina CA, Ribo M et al (2004) Impact of admission hyperglycemia on stroke outcome after thrombolysis: risk stratification in relation to time to reperfusion. Stroke 35:2493-2498

16. Rosso C, Attal Y, Deltour S et al (2011) Hyperglycemia and the fate of apparent diffusion coefficient-defined ischemic penumbra. AJNR Am J Neuroradiol 32:852-856

17. De Silva DA, Ebinger M, Christensen S et al (2010) Baseline diabetic status and admission blood glucose were poor prognostic factors in the EPITHET trial. Cerebrovasc Dis 29:14-21

18. Kimura K, Sakamoto Y, Iguchi Y et al (2011) Admission hyperglycemia and serial infarct volume after t-PA therapy in patients with and without early recanalization. J Neurol Sci 307:55-59

19. Bellolio MF, Gilmore RM, Ganti L (2014) Insulin for glycaemic control in acute ischaemic stroke. Cochrane Database Syst Rev CD005346

20. Rosso C, Corvol JC, Pires C et al (2012) Intensive versus subcutaneous insulin in patients with hyperacute stroke: results from the randomized INSULINFARCT trial. Stroke 43:2343-2349

21. Sandercock P, Wardlaw JM, Lindley RI et al (2012) The benefits and harms of intravenous thrombolysis with recombinant tissue plasminogen activator within $6 \mathrm{~h}$ of acute ischaemic stroke (the third international stroke trial [IST-3]): a randomised controlled trial. Lancet 379:2352-2363

22. Hill MD, Goyal M, Demchuk AM, Fisher M (2015) Ischemic stroke tissue-window in the new era of endovascular treatment. Stroke 46:2332-2334

23. Fan X, Ning M, Lo EH, Wang X (2013) Early insulin glycemic control combined with tPA thrombolysis reduces acute brain tissue damages in a focal embolic stroke model of diabetic rats. Stroke 44:255-259

24. Robbins NM, Swanson RA (2014) Opposing effects of glucose on stroke and reperfusion injury: acidosis, oxidative stress, and energy metabolism. Stroke 45:1881-1886

25. McCormick M, Hadley D, McLean JR, Macfarlane JA, Condon B, Muir KW (2010) Randomized, controlled trial of insulin for acute poststroke hyperglycemia. Ann Neurol 67:570-578 